\title{
Escherichia coli Nissle 1917 engineered to express Tum-5 can restrain murine melanoma growth
}

\author{
Lian He ${ }^{1}$, Huijun Yang ${ }^{1}$, Fei Liü ${ }^{1}$, Yiyan Chen ${ }^{1}$, Sijia Tang ${ }^{1}$, Wei Ji ${ }^{1}$, Jianli Tang ${ }^{1}$, \\ Zhudong Liu ${ }^{1}$, Yunjun Sun ${ }^{1}$, Shengbiao Hu${ }^{1}$, Youming Zhang ${ }^{1}$, Xiong Liu ${ }^{1}$, Weitao \\ Huang $^{1}$, Xuezhi Ding ${ }^{1}$ and Liqiu Xia ${ }^{1}$ \\ ${ }^{1}$ Hunan Provincial Key Laboratory of Microbial Molecular Biology, State Key Laboratory of Developmental Biology of Freshwater \\ Fish, College of Life Science, Hunan Normal University, Changsha 410081, Hunan, China \\ Correspondence to: Liqiu Xia, email: xialq@hunnu.edu.cn \\ Keywords: Tum-5, E. coli Nissle 1917, lux operon, anti-angiogenesis, murine melanoma \\ Received: May 16, $2017 \quad$ Accepted: July 25, $2017 \quad$ Published: August 24, 2017 \\ Copyright: He et al. This is an open-access article distributed under the terms of the Creative Commons Attribution License 3.0 (CC \\ BY 3.0), which permits unrestricted use, distribution, and reproduction in any medium, provided the original author and source are \\ credited.
}

\section{ABSTRACT}

Tumor growth and metastasis depend on angiogenesis. Thus, inhibiting tumor angiogenesis has become promising cancer therapeutic strategy in recent years. Tumstatin is a more powerful angiogenesis inhibitor than endostatin. Anti-angiogenic active fragment encoding amino acids 45-132 (Tum-5) of tumstatin was subcloned into four different inducible expression vectors and successfully solubly expressed in Escherichia coli BL21 (DE3) in this study. Subsequently, an anaerobic inducible expression vector was constructed under Vitreoscilla hemoglobin gene promoter Pvhb in $E$. coli Nissle 1917 ( $\mathrm{ECN}$ ). The secretory expression of Tum-5 in the engineered bacterium was determined in vitro and in vivo by Western blot or immunochemistry. The anti-tumor effect detection demonstrated that EcN could specifically colonize the tumor, and B16 melanoma tumor growth was remarkably restrained by EcN (Tum5 ) in mice bearing B16 melanoma tumor. Abundant infiltrating inflammatory cells were observed in tumor areas of the EcN-treated group through hematoxylin and eosin staining, with a relatively reduced expression of endothelial marker platelet endothelial cell adhesion molecule-1 (PECAM-1/CD31) by immunofluorescence in tumor sections of EcN (Tum-5)-treated mice. No significant morphological differences were observed in the liver, kidney and spleen between EcN-treated mice and the control group, indicating that ECN was cleared by the immune system and did not cause systemic toxicity in mice. These findings demonstrated that the gene delivery of Tum-5 to solid tumors could be an effective strategy for cancer therapy.

\section{INTRODUCTION}

The use of bacteria for cancer treatment has a long history [1-3]. The detailed mechanisms of their clinical effects are still unknown, but previous studies clearly showed that some bacteria could play important roles in treating some diseases, especially cancer. Over the last century, many genera of bacteria, including Salmonella [4, 5, 27], Escherichia [6, 7], Clostridium [8-10], Bifidobacterium [1113] and Listeria [14, 15], have been shown to preferentially accumulate in hypoxic tumor areas. For nearly a decade, bacteria were used in cancer therapy by combining with chemotherapeutic drugs [38, 39] or delivering anti-tumor agents such as interfering RNAs [40, 42] prodrug activating enzymes [30] and cytokines [41, 43] into tumor tissues.

$\mathrm{EcN}$ was first isolated by the army surgeon Dr. Alfred Nissle in 1917 from the feces of a soldier who did not develop diarrhea during a severe outbreak of shigellosis [25]. Intestinal probiotics $\mathrm{EcN}$ have been licensed as a pharmaceutical in several countries for the treatment of diseases, such as diarrhea and colitis ulcerosa, affecting the digestive tract $[16,26]$. Previous research revealed that 
EcN selectively colonized and replicated in the necrotic tumor tissue [7, 17, 28, 29]. Yunlei et al [29] used EcN as a delivery system for gene therapy on tumor-bearing mice. They sacrificed mice on 1, 3, 5, and 7 days after i.v. administration and cultured the lung, liver, spleen, kidney, heart, and tumor tissues. Then, the group found that EcN could only specifically target the tumor tissue. Jochen et al [17] injected EcN intravenously (i.v.), intraperitoneally (i.p.), or intratumorally (i.t.) into tumor-bearing mice to study its specific targeting property. They found that massive $\mathrm{EcN}$ colonized and replicated in tumors and no obvious difference was observed in the $\mathrm{CFU} / \mathrm{g}$ isolated from organ tissues, regardless of inoculation route. The colonization of spleen and liver were significantly lower when EcN strains were used compared with S. typhimurium, and the non-pathogenic strains did not colonize the organs at all. Given its high tumor-selective replication and efficient clearance from the spleen and liver tissues, EcN was selected as a vector to specifically express Tum-5 in further studies.

Tumstatin, the NC1 domain fragment of the type IV collagen alpha3 chain, was deemed as a preferable tumorspecific angiogenesis inhibitor over endostatin. Tumstatin bound to av $\beta 3$ integrin and inhibited the growth of tumors in mouse models $[18,19]$. The anti-angiogenic activity of tumstatin was located in the 54-132 amino acid region (Tum-5) through deletion mutagenesis [19]. This antiangiogenic region was separate from the 185-203 amino acid region responsible for the anti-tumor activity. Tum5 exerted the equal anti-angiogenesis effects similar to that induced by full-length tumstatin. This region bound predominantly to the $\beta 3$ subunit of av $\beta 3$ integrin, and then inhibited the FAK, PI3K, and PKB cell signal transduction pathways, as well as reduced the phosphorylation of the mTOR kinase and eukaryotic initiation 4E. The latter effect led to the blocking of endothelial cell protein synthesis. As a result, the formation of new blood vessels were suppressed [20]. Finally, tumor growth, infiltration, and metastasis were suppressed after the up-regulation of Tum-5 expression.

In this study, the anti-tumor effect of EcN (Tum5) on the mice bearing B16 mouse melanoma has been investigated in vivo. The engineered bacterium efficiently suppressed the growth of tumors by releasing Tum-5 protein and inhibiting tumor angiogenesis in vivo. This work laid an important foundation for bacteria-mediated tumor therapy.

\section{RESULTS}

\section{Expression analysis of Tum-5 protein in vitro}

To solubly express Tum-5 in prokaryotic expression system, the Tum-5 gene was cloned into four inducible expression vectors with different labels (Supplementary Figure 1A and 1B). Western blot and mass spectrometry analysis demonstrated that the Tum-5 protein was successfully expressed in E. coli BL21 (DE3) (Supplementary Figure 1C and 1D). Coomassie Brilliant
Blue staining analysis showed the recombinant proteins were souble expressed in the presence of SUMO and IF2 tag (Figure 1A). The continuous secretion of Tum- 5 by the engineered bacterium is important in eliciting its anti-tumor activity. Therefore, Tum-5 was coexpressed with the pelB leader sequence under the oxygen-dependent promoter of the hemoglobin gene ( $v h b)$ of Vitreoscilla (Figure 1B and Supplementary Figure 2). The bacterial cells were cultivated in LB medium overnight and harvested by centrifugation. SDS-PAGE results confirmed that the Tum-5 protein was successfully expressed in EcN (Figure 1C). Western blot indicated that the Tum-5 protein was present in both cell lysate and medium supernatant of the EcN (Tum-5) (Figure 1D). The presence of two bands in Western blot may correspond to the presence of the pelB signal peptide before and after resection, because the gap between molecular sizes of these two bands is exactly equal to the size of pelB. The molecular weight of the SUMO tag was lower than that of the IF2 tag. Thus, the SUMO fusion system was selected to investigate the activity of the Tum-5 protein in the subsequent experiments.

\section{EcN specifically colonized and delivered Tum-5 to solid tumors}

IVIS can be used to accurately observe the real-time distribution of bacteria in the animal body without any side effects $[28,35]$. The colonization of $\mathrm{EcN}$ was investigated in mice bearing B16 melanoma at different time points after i.p. injecting $5 \times 10^{6} \mathrm{CFU} / 100 \mu \mathrm{l} \mathrm{EcN} \mathrm{(Lux).} \mathrm{The}$ fluorescence signal was detected immediately at the injection site after intraperitoneal injection (Figure 2A). On the first day, the bacteria spread throughout all organs and tumors in the mice (Figure $2 \mathrm{~A}$ and $2 \mathrm{~B}$ ). This result confirms that the animals have started to eliminate bacteria through their own immune system. Over the following 3-7 days, the bacteria steadily accumulated in tumors and were gradually removed from other organs. This finding implies that the bacteria require an adaptive process to specifically target the tumor (Figure 2A and 2B). Importantly, some mice possessed powerful immune systems and completely removed the bacteria from the liver, kidney, and spleen in 3-5 days without affecting the EcN-targeting tumors (data not shown). These discovery confirms that EcN exhibited excellent tumor-targeting properties.

To ensure that Tum-5 was successfully expressed in tumor areas to inhibit angiogenesis, we performed immunohistochemistry on each group of mouse tumors. The tumor-bearing mice were injected with sterilized PBS or $5 \times 10^{6} \mathrm{CFU} / 100 \mu \mathrm{l}$ bacteria. The tumor tissues were fixed in $4 \%$ paraformaldehyde and then embedded in paraffin after the mice were killed. Immunohistochemistry was used to determine whether Tum-5 could be expressed in tumor tissue. A significant gray signal was obviously displayed in the tumor section of the EcN (Tum-5)treated group. However, the other groups did not display a 
positive signal (Figure 2C). The results demonstrated that Tum-5 was successfully expressed in the tumor region.

\section{EcN (Tum-5) significantly inhibited B16F10 melanoma proliferation in $\mathrm{C} 57 \mathrm{BL} / 6$ mice}

The in vivo anti-tumor activity of Tum-5 in xenograft mice with B16F10 melanoma cells was assessed (Figure 3). B16 melanoma cells were implanted into the right axillary subcutaneous area of the C57BL/6 mice. The resultant model was then used for investigating the anti-tumor effects of EcN (Tum-5). When the tumor volume reached approximately $60 \mathrm{~mm}^{3}$, the mice were randomly divided into four groups $(n=6,7,8)$. Each group was injected with sterilized PBS or $5 \times 10^{6}$ CFU/100 $\mu$ l bacteria. The growth of the EcN (Tum-5)treated tumors was remarkably suppressed. Compared with the mice in the PBS-, EcN-, and EcN (28a)-treated groups, the transplanted tumors of the EcN (Tum-5)treated groups grew slower (Figure 4A). However, the groups that received $\mathrm{EcN}$ or $\mathrm{EcN}$ (28a) alone did not differ from the PBS group in terms of tumor suppression. The tumor weight of the EcN (Tum-5)-treated mice was significantly lower than those of the other groups (Figure 4B). The tumor volume of the PBS group reached up to $6813.66 \pm 1021.38 \mathrm{~mm}^{3}$, but the tumor volume was significantly repressed by EcN (Tum-5), with a volume of $3205.83 \pm 550.46 \mathrm{~mm}^{3}$. The tumor growth was inhibited by approximately $52.95 \%$ with respect to that of the PBS group $(P<0.01)$ based on the formula (Table 1$)$.

\section{Histological morphology and MVD of the tumor tissues}

Through H\&E staining, the solid tumor sections generally showed enlarged tumor cells and nuclei. Nuclear chromatin was purple-blue under the hematoxylin stain, and the cytoplasm was stained red by eosin. Tumor tissue developed cell shrinkage and chromosome degradation when the cells were apoptotic [21, 22]. The morphological change of the tumor tissue in the tumor-burdened mice was observed after H\&E staining. In the PBS-treated

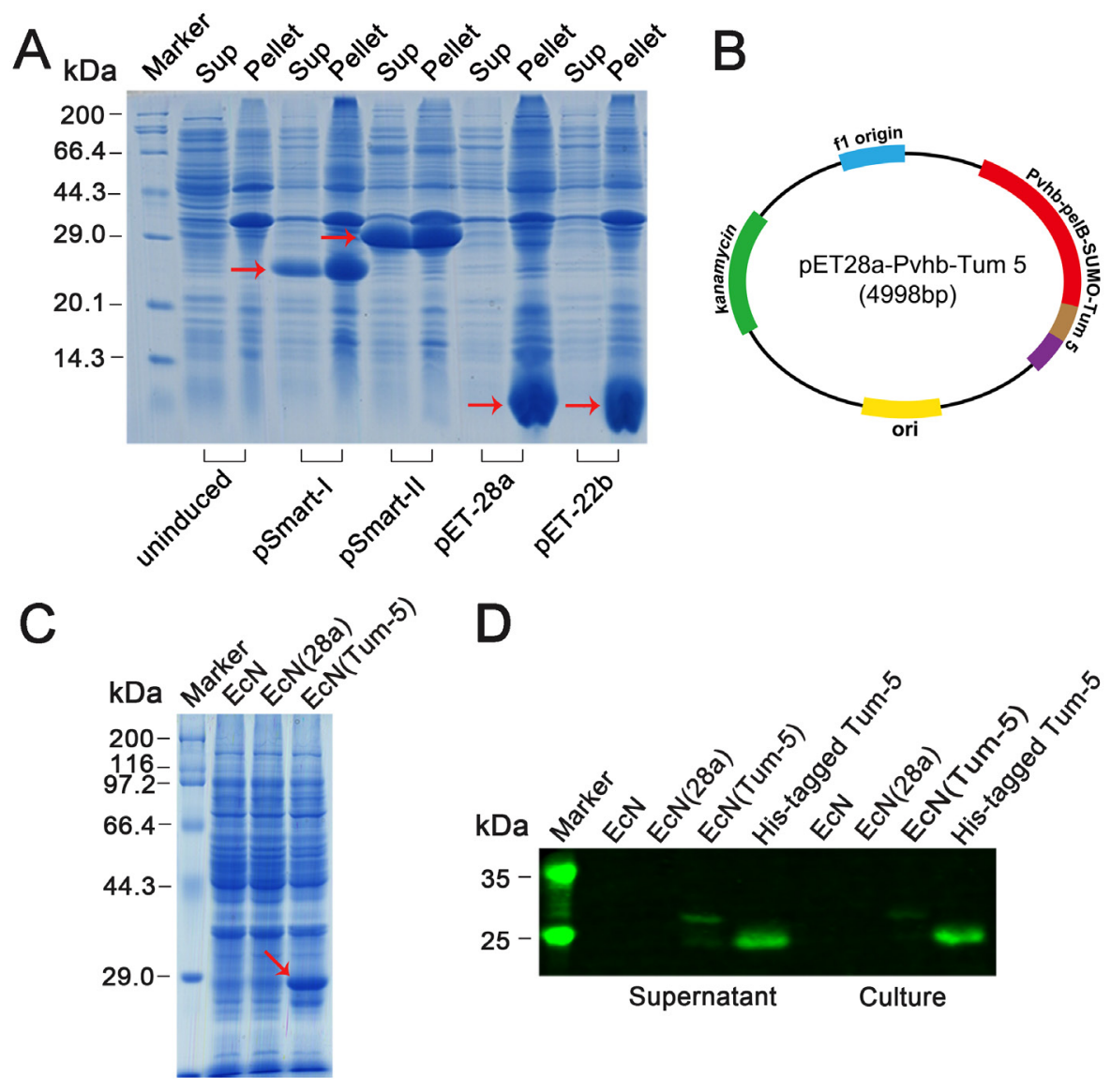

Figure 1: Characterization of Tum-5 expression in vitro. (A) Coomassie Brilliant Blue staining of SDS-PAGE showing Tum-5 expression in E.coli BL21 (DE3). (B) Map of recombination plasmid containing Tum-5 gene. (C) SDS-PAGE analysis of Tum-5 expression in $\mathrm{EcN}, \mathrm{EcN}$ (28a), and $\mathrm{EcN}$ (Tum-5). (D) Western blot analysis of Tum-5 expression in cell lysates (supernatants) or culture supernatants of $\mathrm{EcN}, \mathrm{EcN}(28 \mathrm{a})$, and $\mathrm{EcN}$ (Tum-5). 

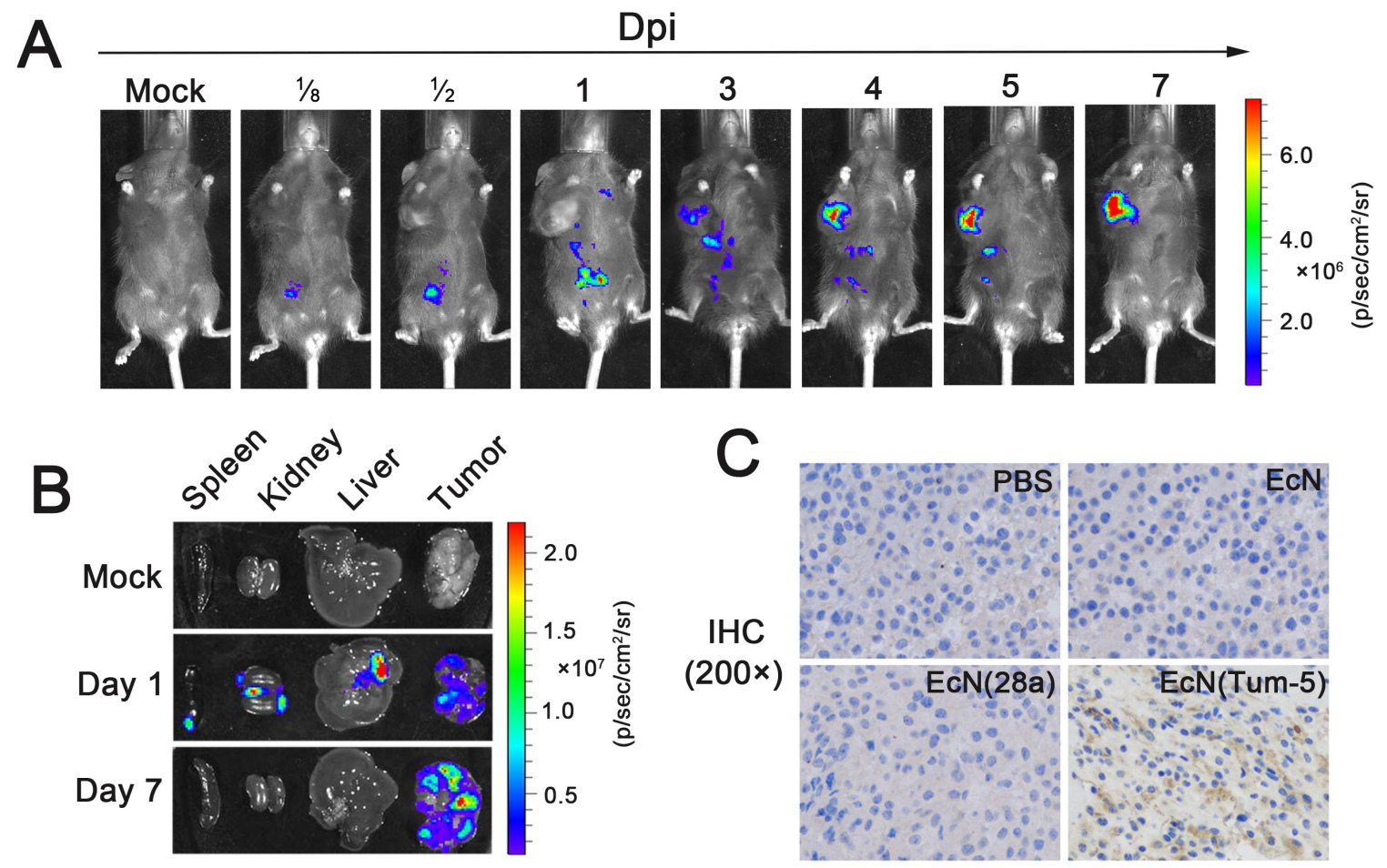

Figure 2: Colonization profile of EcN in the murine B16 tumor model and characterization of Tum-5 expression in vivo. $1 \times 10^{5} \mathrm{~B} 16 \mathrm{~F} 10$ cancer cells were inoculated into the right axillary subcutaneous of $\mathrm{C} 57 \mathrm{BL} / 6$ mice. After $7-10$ days, the mice were injected with $5 \times 10^{6} \mathrm{CFU} / 100 \mu \mathrm{EcN}$ (Lux). (A) The distribution of the bacteria in the tumor-bearing mice were detected using IVIS. (B) The tumor, liver, kidney, and spleen of the mice were isolated at different times and observed using IVIS. (C) Tum-5 was confirmed to be successfully expressed in the tumor tissue by IHC $(200 \times)$ using its specific antibody. Almost no positive signal was observed in tumor sections in the PBS-, EcN-, EcN (28a)-treated mice. "Dpi" means "days post infection."
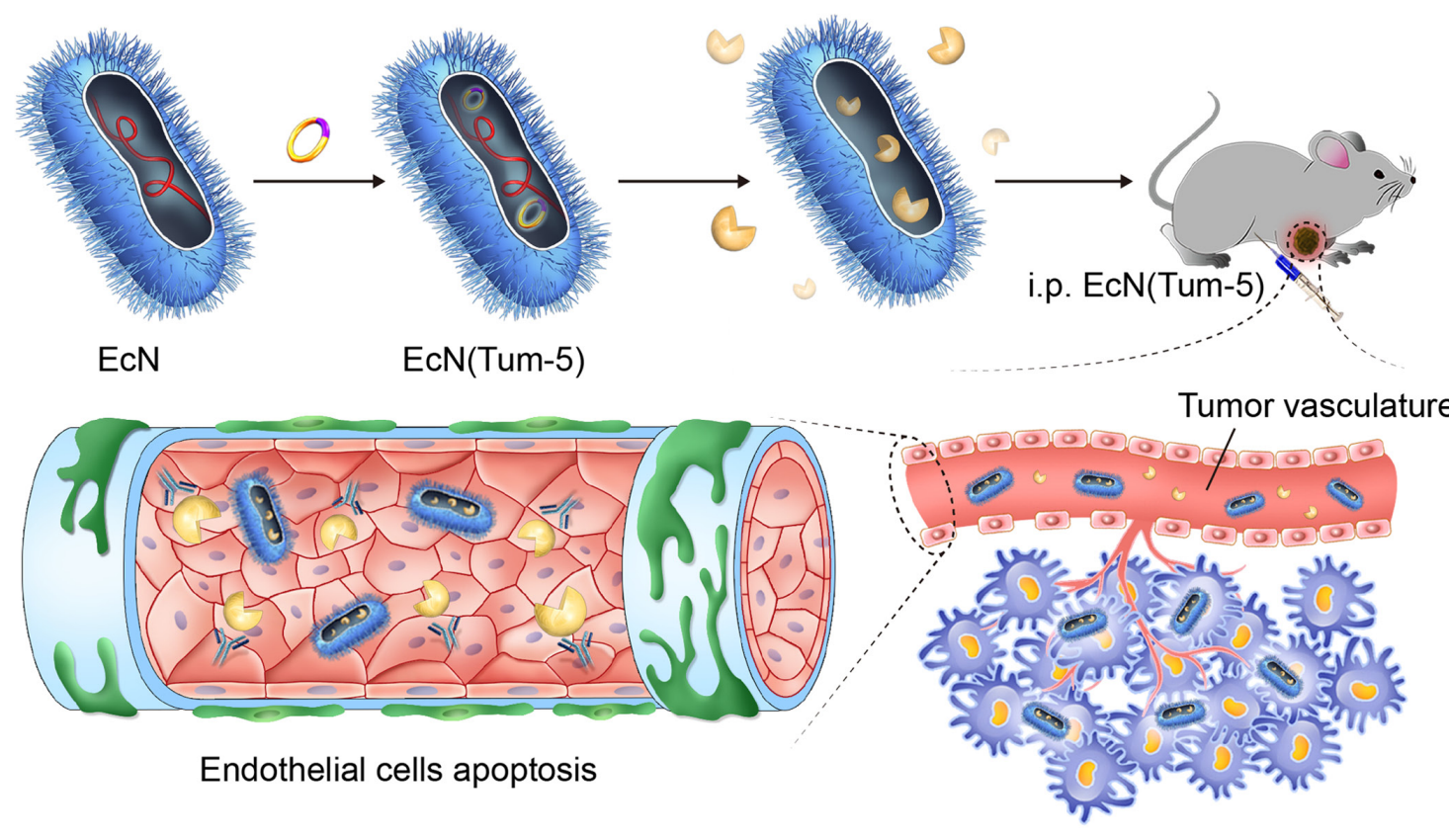

Figure 3: EcN (Tum-5) probiotics for cancer therapy. Tum-5 protein (yellow) could be soluby expressed in EcN and secreted to the medium. The engineered bacteria (blue) were rapidly specially colonized in mouse tumors. Tum-5 bound to integrin receptors on the surface of vascular endothelial cells to induce endothelial cell apoptosis. This process would cause blood vessels to shrink, then the tumor growth was suppressed. 
Table 1: The comparison of tumor volume, tumor weight of B16F10 cancer of the C57BL/6 mice

\begin{tabular}{lcc}
\hline Group & $\begin{array}{c}\text { Mean tumor } \\
\text { volume }\left(\mathbf{m m}^{\mathbf{3}}\right)\end{array}$ & $\begin{array}{c}\text { Mean tumor } \\
\text { weight (g) }\end{array}$ \\
\hline PBS & $6813.66 \pm 1021.38$ & $5.76 \pm 1.15$ \\
EcN & $5930 \pm 591.14$ & $5.17 \pm 1.06$ \\
EcN (28a) & $6481 \pm 609.53$ & $5.09 \pm 1.02$ \\
EcN (Tum-5) & $3205.83 \pm 550.46(52.95 \%)^{* *}$ & $2.97 \pm 1.15(48.43 \%)^{*}$ \\
\hline
\end{tabular}

groups, tumor tissue exhibited a fairly complete structure and regular shapes. Moreover, no necrotic area was observed. However, massive infiltrating inflammatory cells (red arrow) were observed in the EcN-treated group (Figure 5A). No significant change in the histopathological morphology of organs were observed between the PBS and EcN groups throughout the experiment (Figure 5A). This result implies the negligible side effect on the liver, kidney, and spleen. The endothelial marker PECAM-1/CD31 is expressed in the endothelium of both lymphatic and blood vessels [23, 24]. Immunofluorescence assay revealed that the expression of CD31 and the microvessel density in the EcN (Tum-5)-treated group were significantly lower than those in the other three groups (Figure 5B). These findings illustrated that EcN (Tum-5) achieved an eminent anti-tumor activity, which might be due to the antiangiogenic effect of the Tum-5 protein. Therefore, the anti-angiogenesis effect of Tum-5 might suppress tumor angiogenesis and indirectly inhibit the growth, infiltration, and metastasis of tumors.

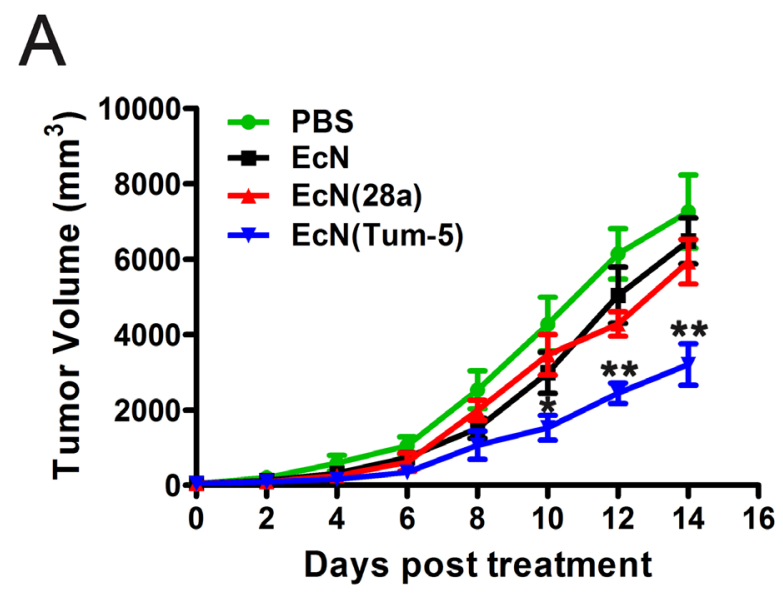

\section{Safety monitoring of $E$. coli Nissle 1917}

EcN toxicity in tumor-bearing $\mathrm{C} 57 \mathrm{BL} / 6$ mice $(\mathrm{n}=6$, $7,8)$ was evaluated every 6 days after $5 \times 10^{6} \mathrm{CFU} / 100 \mu \mathrm{l}$ $\mathrm{EcN}$ was administered. Body weight was measured every 2 days during treatment. At the end of the experiment, the liver, kidney, and spleen were excised and weighed. The organs of EcN-treated mice were not significantly differ from those of mice in PBS groups (Figure 6A). Although the body weight of the mice decreased slightly after injection, the weight immediately returned to normal in the next 2 days (Figure 6B). In general, these results demonstrated that the systemic administration of EcN did not substantially exert adverse effects on animals.

\section{DISCUSSION}

In this study, the anti-tumor effect of $\mathrm{EcN}$ was assessed as a new drug carrier to deliver Tum-5 for cancer therapy. Tum-5 inhibited tumor growth, and this inhibitory

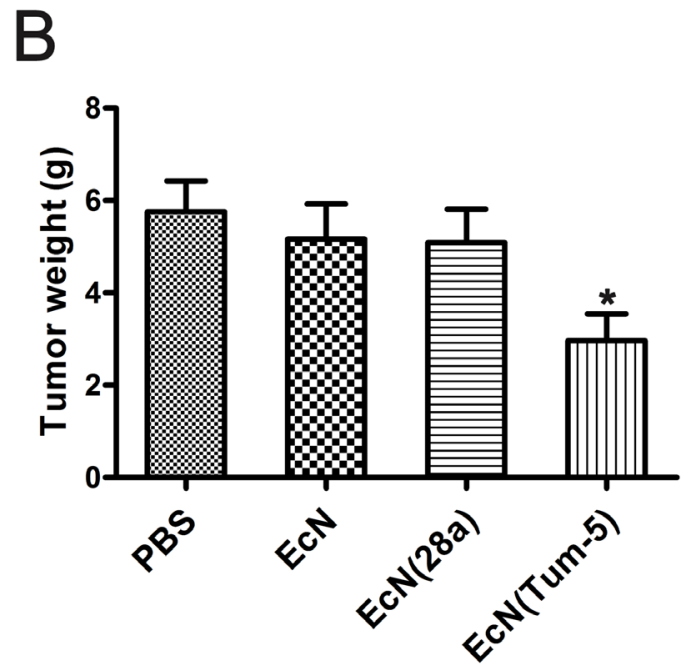

Figure 4: Inhibitory effect of EcN (Tum-5) on B16F10 melanoma tumor growth. Therapeutic effect of EcN (Tum-5) on mice $(\mathrm{n}=6,7,8)$ bearing B16 melanoma tumors. Mice were treated by i.p. administration of either PBS or $5 \times 10^{6} \mathrm{CFU} / 100 \mu 1$ of EcN, EcN $(28 \mathrm{a})$, or EcN (Tum-5) at every 6-day intervals. Tumor volumes $\left(\mathrm{mm}^{3}\right)$ were estimated using external calipers (values are expressed as means \pm standard deviations [SDs]) (A). B16 melanoma tumors were significantly inhibited by EcN (Tum-5) compared with the PBS control group. Mice were sacrificed, and tumor weights were measured (B). 

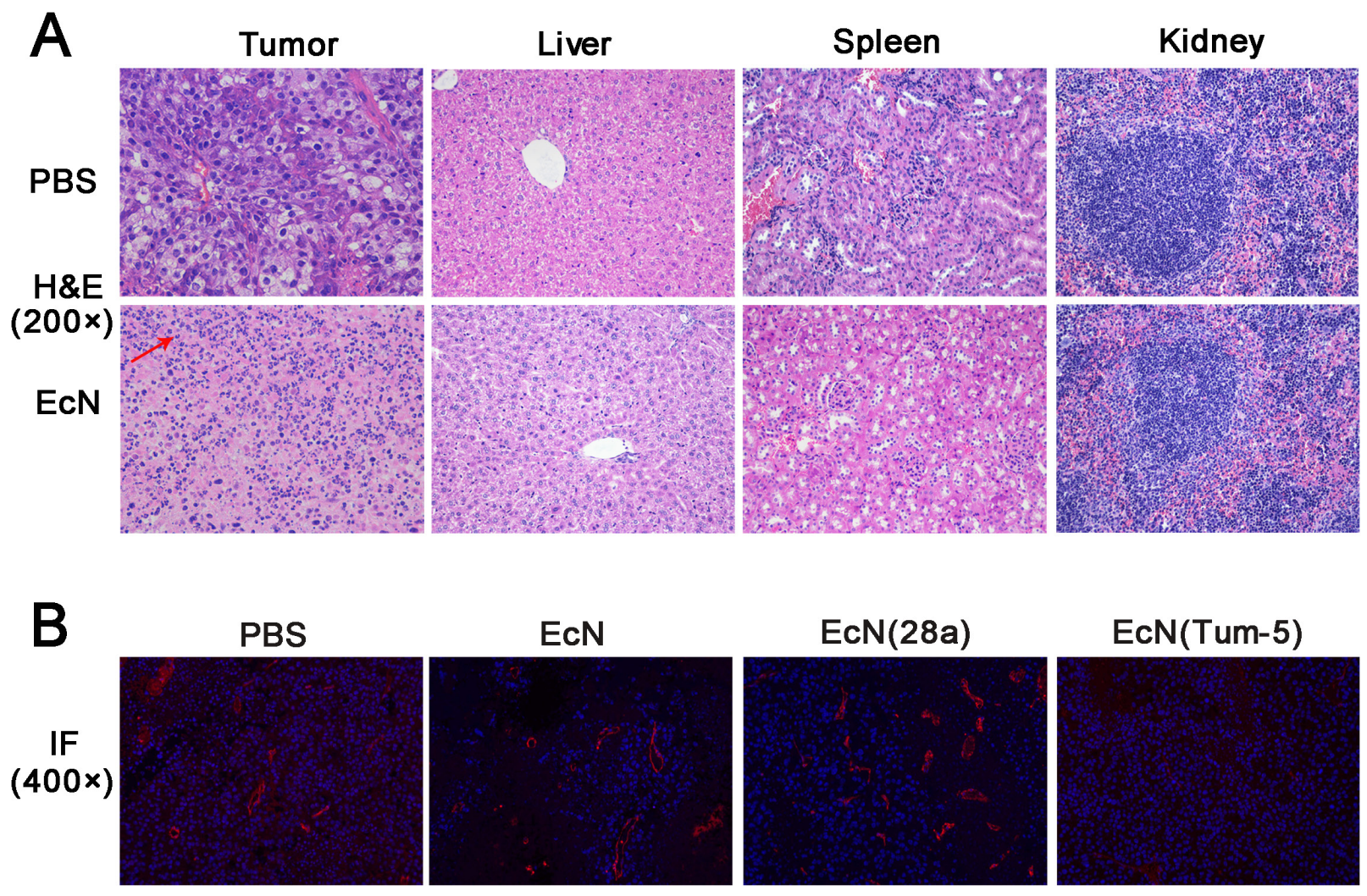

Figure 5: HE staining of tumor tissue sections and immunofluorescence of CD31 expression in vivo. H\&E staining $(400 \times)$ of the tumor sections was used to observe biopsy pathological morphology (A). IF staining (200×) for DAPI-stained nuclei (blue), CD31 (red) were evaluated by fluorescence microscopy (B). Positive signals were hardly observed in the tumor sections of EcN (Tum-5)-treated mice.

A
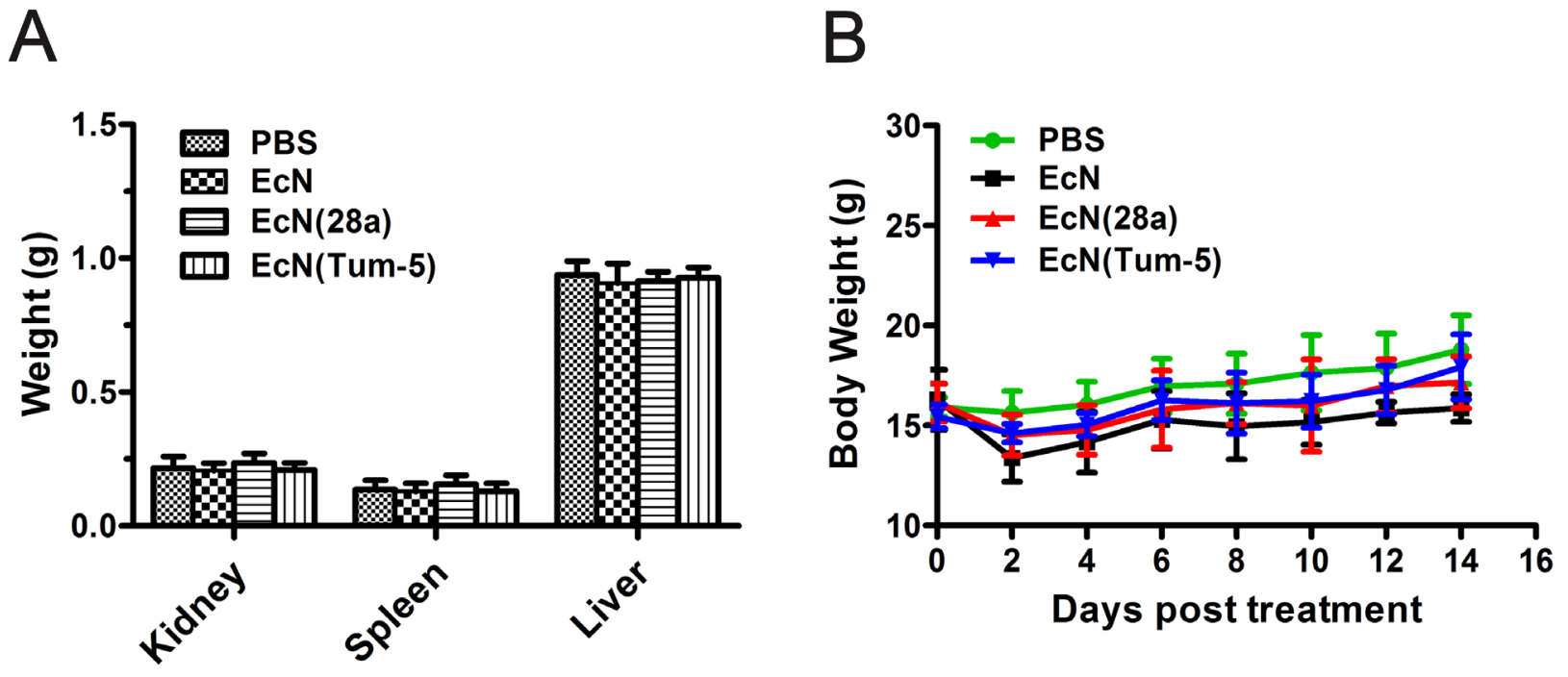

Figure 6: Body weight and kidney, spleen, and liver weight changes of mice before or after bacterial treatment. Mice bearing B16 mouse melanoma were injected every 6 days with $5 \times 10^{6} \mathrm{CFU} / 100 \mu 1 \mathrm{EcN}, \mathrm{EcN}(28 \mathrm{a})$, EcN (Tum-5), or sterilized PBS three times. At the end of the experiment, the kidney, liver, and spleen were excised and weighed (means and SDs) (A). The body weights were recorded at 2-day intervals. (B) No significant difference was observed between the PBS and EcN treatment groups. 
effect was likely due to the transgenic expression of Tum5 and the delivery of bacterial EcN, because the control groups failed to exhibit similar effects.

At present, drugs are administered to experimental animals through intravenous injection, intraperitoneal injection, and gavage. Intraperitoneal administration has its own unique advantages and has been successfully used in the treatment of multiple tumor models [45, 46, 47]. The peritoneum dense blood vessels and lymphatic vessels, thus allowing for the strong absorption capacity of solutes without overloading the heart. Solutes vacate the peritoneal cavity into the systemic circulation either by diffusion through the parietal/visceral peritoneum or by absorption through lymphatic tissue. The plasma-peritoneal barrier has unidirectional transport characteristics, with intraperitoneally administered substances appearing rapidly in systemic circulation, and with intravenously administered substances appearing slowly in peritoneal fluid. In a phase-I trial by Vasey et al [46], adenovirus d11520 was intraperitoneally administered in patients with recurrent ovarian cancer. Tsutomu et al [47] intraperitoneally administered docetaxel to treat peritoneally disseminated cancer. Their results indicated that the intraperitoneal administration of docetaxel is likely an effective treatment method for peritoneally disseminated cancer. In addition, this method is not associated with increase systemic toxicity. Furthermore, given that intraperitoneally injected bacteria could migrate to regional lymph nodes, mesentery, and other tissues, the anti-angiogenic Tum- 5 protein might be able to decrease tumor metastases to the lymph nodes and other organs.

The bacterial colonization of tumors was initially attributed to the hypoxic nature of solid tumors. The involvement of bacterial chemotaxis toward chemoattractant compounds (e.g., aspartate, serine, citrate, ribose, or galactose) in necrotic regions of quiescent cancer cells has also been suggested as a contributing factor [44]. In this study, the mice were dissected to isolate the tumor, liver, kidney, and spleen. Then, the IVIS system was used to observe the distribution of bacteria in these organs after intraperitoneal injection. Although the bacteria were systemically distributed within a short period of time, the bacteria in other organs were removed by the immune system over time. Thus, EcN achieved an excellent targeting property to the anaerobic environment of tumor tissues, and the present experimental results also confirmed this view. These findings suggest that the intraperitoneal injection of bacteria also allowed the bacteria to specifically colonize in the tumors of the tumor-bearing mice. In addition, immunohistochemistry confirmed that Tum-5 was efficiently and consecutively expressed in the tumor tissues. These results confirmed that EcN could colonize the solid tumor areas. These findings thus lay an important foundation for future studies on other tumor-targeting bacteria.
As the anti-angiogenic active region of tumstatin, Tum-5 has been studied since its discovery $[18,19,31$, 32]. Li et al [33] placed Tum-5 in the pLXSN retroviral vector and found that the pLXSN-Tum-5 significantly inhibited the growth of human umbilical vein endothelial cells and H22 HCC cell tumor. Yanjie et al [34] confirmed that Tum-5 could inhibit the growth of S180 tumors in tumor-bearing mice. In the present study, the antiangiogenic effects of Tum-5 and the anti-tumor effects exerted by $\mathrm{EcN}$ (Tum-5) in vivo were investigated using B16 melanoma tumor cells. The tumor volume and weight were significantly repressed by EcN (Tum$5)$. Notably, the tumor growth was extremely restrained by EcN (Tum-5), which led to $52.95 \%(P<0.01)$ and $48.43 \%(P<0.05)$ repression on tumor volume and tumor weight, respectively. The present research indicated that EcN (Tum-5) significantly inhibited the growth of B16 melanoma, whereas the body and tissue weight of mice were not affected.

Tumor angiogenesis is a critical feature of tumor growth, and some anti-angiogenesis agents were developed to cure tumor by blocking tumor angiogenesis $[36,37]$. Immunofluorescence analysis with CD31 antibody indicated that the average microvessel density in the EcN (Tum-5) group was significantly lower than that in the PBS and EcN groups. These results suggested that Tum-5 exerted its anti-tumor activity by suppressing vascular endothelial cells. Whether EcN (Tum-5) can inhibit the angiogenesis of other tumor cells in tumorbearing mice must be explored. Combining EcN (Tum5) with radiotherapy, chemotherapy, or other cancer treatments also merits further study.

Interestingly, the body weight of each mouse group decreased slightly after injection but immediately returned to normal in the next 2 days for the entire treatment experiment. This result maybe the normal response of the mice to treatment, because the mouse weights in each group were stable throughout the treatment courses [7, 21]. In general, the PBS-treated mice held a slight advantage in body weight. Although the tumor volume was increasing, the mice became increasingly thin due to aggravating illness. As a result, the mouse weight did not show a significant upward trend. The mice in the EcN (Tum-5)treated group exhibited improved physical fitness after the tumor volume was reduced by the therapeutic effect of Tum-5. Accordingly, no significant difference in weight was observed between the EcN (Tum-5)- and PBS-treated mice. The mice in the EcN group and the EcN (28a) group were similar to those in the PBS group. Therefore, no significant difference in body weight was observed among the four groups, and the results were reasonable.

In summary, bacteria engineered for Tum-5 gene delivery was successfully constructed. These bacteria were designed to specifically deliver gene to the tumor area and exert the gene's anti-angiogenesis efficacy. Using tumor-targeting bacteria EcN to deliver therapeutic agents 
to solid tumors could serve as a prospective approach in future tumor therapy.

\section{MATERIALS AND METHODS}

\section{Animal and cell culture}

All animal experiments followed the National Institutes of Health Guide for the Care and Use of Laboratory Animals and were approved by the Animal Ethics Committee of Hunan Normal University. C57BL/6 mice (6-8 weeks) were fed under specific pathogen-free (SPF) conditions. B16F10 melanoma cells were cultured at $37{ }^{\circ} \mathrm{C}$ and in $5 \% \mathrm{CO}_{2}$ atmosphere in 1640 supplemented with $10 \%$ heat-inactivated fetal bovine serum containing $100 \mathrm{U} / \mathrm{ml}$ penicillin and $100 \mu \mathrm{g} / \mathrm{ml}$ streptomycin.

\section{Gene cloning and soluble expression of Tum-5}

DNA sequences of Tum-5 (amino acids 45-132 of tumstatin) were obtained by spliced overlap extension PCR technology. The primers used in the study were listed in Supplementary Table 1. The Tum-5 gene was inserted into the Nco I and Xho I restriction enzyme sites of pET28a and pET-22b. Tum-5 was cloned into BamH I and Xho I restriction sites of pSmart-I (small ubiquitin-related modifier-SUMO fusion expression system) and pSmartII (initiation factor-IF2 protein structure domain I fusion expression system). The resulting vectors were named pET-28a-Tum 5, pET-22b-Tum 5, pSmart-I-Tum 5, and pSmart-II-Tum 5. The four plasmids were transformed into E. coli BL21(DE3) and cultured at $37^{\circ} \mathrm{C}$ in LB medium supplemented with $100 \mu \mathrm{g} / \mathrm{ml}$ ampicillin or $50 \mu \mathrm{g} / \mathrm{ml}$ kanamycin. Until the $\mathrm{OD}_{600}$ value reached 0.4-0.6, IPTG was added at a final concentration of $0.3 \mathrm{mM}$ to induce Tum-5 protein expression at $30^{\circ} \mathrm{C}$. Cells were harvested by centrifugation at $8000 \mathrm{rpm}$ after $4 \mathrm{~h}$ induction. After washed twice and resuspended in a mixture of $50 \mathrm{mM}$ $\mathrm{NaH}_{2} \mathrm{PO}_{4}$ and $300 \mathrm{mM} \mathrm{NaCl}$ at $\mathrm{pH} 8.0$, the cells were lysed by sonication, and the supernatants and pellets were analyzed by sodium dodecyl sulfate-polyacrylamide gel electrophoresis (SDS-PAGE) after centrifugation. The recombinant protein was cut with a surgical blade and identified by LTQ XL mass spectrometry (Thermo Fisher) after proteolysis.

\section{Construction of EcN expression strains}

The Vitreoscilla hemoglobin gene promoter Pvhb was amplified from pET-28a-Pvhb-pelB-asp (Lab store). Meanwhile, the Sumo-Tum 5 fragment was amplified from pSmart-I-Tum 5 (constructed in this study). Pvhb-pelBSUMO-Tum 5 was obtained by overlap extension PCR and inserted into pET-28a after digestion by Apa I and Xho I. The sequenced vector was transformed into EcN by electroporation and named EcN (Tum-5). pET-28a was also transformed into $\mathrm{EcN}$ as negative control and named $\mathrm{EcN}$ (28a). EcN (Tum-5), EcN (28a) and EcN were cultured in LB medium for $10 \mathrm{~h}$. Then, Western blot was used to confirm the expression of Tum- 5 in both cell supernatant and culture supernatant by using Anti- $6 \times$ His rabbit polyclonal antibody. The medium supernatant was precipitated with acetone containing 10\% TCA and then washed with $90 \%$ acetone. Total cell protein $(10 \mu \mathrm{g})$ was separated through SDS-PAGE and transferred to polyvinylidene difluoride membranes (Millipore) using a semidry method. The result was detected by IRDye ${ }^{\circledR} 680$ conjugated goat anti-rabbit IgG (LI-COR, America) and observed by Odyssey Infrared Imaging System (LI-COR, America).

\section{Animal experiments}

C57BL/6 female mice (6-8 weeks old) were purchased from the SLRC Laboratory Animal Company (Hunan, China). The animals were bred and maintained under SPF conditions for at least 3 days before use. For the melanoma tumor model, $1 \times 10^{5} \mathrm{~B} 16 \mathrm{~F} 10$ cancer cells suspended in $100 \mu \mathrm{l}$ PBS were injected into the right axillary subcutaneous of the C57BL/6 mice.

After the tumor volume reached $60 \mathrm{~mm}^{3}$, the mice were divided into four groups (6-8 mice per group) randomly. The mice were intraperitoneally (i.p.) injected with sterilized PBS, EcN, EcN (28a) or EcN (Tum-5) every 6 days at three times. The amount of bacteria injected was $5 \times 10^{6}$ colony-forming units $(\mathrm{CFU}) / 100 \mu \mathrm{l}$. The body weights and tumor volumes were measured every 2 days over the whole experiment. Then, all mice were sacrificed for analysis at day 14, and the weights of the tumor, liver, kidney, and spleen were measured at the end of treatment. The anti-tumor activities of the treatments were evaluated by tumor growth inhibition. Tumor volume (TV) was calculated according to the formula: TV $\left(\mathrm{mm}^{3}\right)=\mathrm{d}^{2} \times \mathrm{D} / 2$, where $\mathrm{d}$ and $\mathrm{D}$ are the shortest and the longest diameter, respectively. Tumor suppression percentage was calculated by the following computational formula: (control group - treatment group) / control group $\times 100 \%$ (with tumor volume or tumor weight to calculate).

\section{Non-invasive in vivo imaging}

To monitor bacteria distribution in mice after injection, a vector for the constitutive expression of lux was constructed by inserting the luxCDABE operon from Photorhabdus luminescens into the pET-28a vector. Then, the pET-28a-Lux plasmid was transformed into EcN by electroporation. When the tumor volume has grown to the appropriate size, the mouse was i.p. injected with $5 \times 10^{6}$ CFU/100 $\mu \mathrm{EcN}$ (Lux) to observe the colonization of the bacteria in the living body at different time points using in vivo imaging system (IVIS, Calipers). The mice were anesthetized with $2 \%$ isoflurane by using a XGI- 8 gas system (Calipers). 


\section{Histological morphology and microvessel density (MVD) of tumor}

Upon isolation, tumors were fixed in $4 \%$ paraformaldehyde overnight after the mice were sacrificed and embedded in paraffin. Then, the tumors were prepared for hematoxylin and eosin (H\&E) staining and immunofluorescent (IF) assay in accordance with standard laboratory procedures. DAPI was used to label cell nucleus and endothelial marker CD31 antibody was adopted to mark the microvessels. The specimens were observed and photographed under the microscope or fluorescence microscope. The expression of Tum-5 was assayed by immunohistochemical (IHC) staining using similar approaches. The liver, spleen, and kidney of the PBS group and $\mathrm{EcN}$ groups were also prepared for $\mathrm{H} \& \mathrm{E}$ staining to determine whether $\mathrm{EcN}$ has noticeable toxicity on the animals.

\section{Statistical analysis}

All data were expressed as the mean \pm standard deviation and analyzed using software IBM SPSS statistics 21.0. The statistical significance for all experimental groups was determined by student's $t$-test. A difference with a $P$ value of less than 0.05 was considered statistically significant, whereas $P<0.01$ was considered extremely significant.

\section{Abbreviations}

EcN: Escherichia coli Nissle 1917; PECAM-1/ CD31: endothelial marker platelet endothelial cell adhesion molecule-1; H\&E: hematoxylin and eosin staining; IF: immunofluorescent; IHC: immunohistochemical; IF: initiation factor; i.v.: intravenously; i.p.: intraperitoneally; i.t.: intratumorally; SDS-PAGE: sodium dodecyl sulfate-polyacrylamide gel electrophoresis; SUMO: small ubiquitin-related modifier.

\section{Author contributions}

Conceived the research idea: LH, LQX. Designed the experiments: LH, HJY, YYC, LQX. Methodology: LH, FL, SJT, WJ. Performed the experiments: LH, YYC, SJT, WJ. Discussed the results: LH, JLT, ZDL. Analyzed the data: LH, YJS, SBH. Wrote the manuscript: LH.

Discussed and revised the manuscript: LH, YMZ, XL, WTH, XZD, LQX. All authors read and approved the final manuscript.

\section{CONFLICTS OF INTEREST}

No potential conflicts of interest, including financial and personal interests, is involved in the publication.

\section{FUNDING}

This work was supported by the National Basic Research Program (973) of China (2012CB722301), the National High Technology Research and Development program (863) of China (2011AA10A203), the International Cooperation Project (0102011DFA32610) and the Cooperative Innovation Center of Engineering and New Products for Developmental Biology of Hunan Province (20134486).

\section{REFERENCES}

1. Wiemann B, Starnes CO. Coley's toxins, tumor necrosis factor and cancer research: a historical perspective. Pharmacol Ther. 1994; 64:529-64. https://doi. org/10.1016/0163-7258(94)90023-X.

2. Heppner F, Möse JR. The liquefaction (oncolysis) of malignant gliomas by a non pathogenic Clostridium. Acta Neurochir (Wien). 1978; 42:123-5. https://doi.org/10.1007/ BF01406639.

3. Forbes NS. Engineering the perfect (bacterial) cancer therapy. Nat Rev Cancer. 2010; 10:785-94. https://doi. org/10.1038/nrc2934.

4. Pawelek JM, Low KB, Bermudes D. Tumor-targeted Salmonella as a novel anticancer vector. Cancer Res. 1997; 57:4537-44.

5. Loeffler M, Le'Negrate G, Krajewska M, Reed JC. IL-18producing Salmonella inhibit tumor growth. Cancer Gene Ther. 2008; 15:787-94. https://doi.org/10.1038/cgt.2008.48.

6. Yu YA, Shabahang S, Timiryasova TM, Zhang Q, Beltz R, Gentschev I, Goebel W, Szalay AA. Visualization of tumors and metastases in live animals with bacteria and vaccinia virus encoding light-emitting proteins. Nat Biotechnol. 2004; 22:313-20. https://doi.org/10.1038/nbt937.

7. Kocijancic D, Felgner S, Frahm M, Komoll RM, Iljazovic A, Pawar V, Rohde M, Heise U, Zimmermann K, Gunzer F, Hammer J, Crull K, Leschner S, Weiss S. Therapy of solid tumors using probiotic Symbioflor-2 restraints and potential. Oncotarget. 2016; 7:22605-22. https://doi. org/10.18632/oncotarget.8027.

8. Malmgren RA, Flanigan CC. Localization of the vegetative form of Clostridium tetani in mouse tumor following intravenous spore administration. Cancer Res. 1955; $15: 473-8$

9. Staedtke V, Bai RY, Sun W, Huang J, Kibler KK, Tyler BM, Gallia GL, Kinzler K, Vogelstein B, Zhou S, Riggins GJ. Clostridium novyi-NT can cause regression of orthotopically implanted implanted glioblastomas in rats. Oncotarget. 2015; 6:5536-46. https://doi.org/10.18632/ oncotarget.3627.

10. Park W, Cho S, Huang X, Larson AC, Kim DH. Branched gold nanoparticle coating of Clostridium novyi-NT spores 
for CT-guided intratumoral injection. Small. 2017; 13. https://doi.org/10.1002/smll.201602722.

11. Kohwi Y, Imai K, Tamura Z, Hashimoto Y. Antitumor effect of Bifidobacterium infantis in mice. Gan. 1978; 69:613-8.

12. Yazawa K, Fujimori M, Nakamura T, Sasaki T, Amano J, Kano Y, Taniguchi S. Bifidobacterium longum as a delivery system for gene therapy of chemically induced rat mammary tumors. Breast Cancer Res Treat. 2001; 66:16570. https://doi.org/10.1023/A:1010644217648.

13. Sivan A, Corrales L, Hubert N, Williams JB, AquinoMichaels K, Earley ZM, Benyamin FW, Lei YM, Jabri B, Alegre ML, Chang EB, Gajewski TF. Commensal Bifidobacterium promotes antitumor immunity and facilitates anti-PD-L1 efficacy. Science. 2015; 350:108489. https://doi.org/10.1126/science.aac4255.

14. Maciag PC, Radulovic S, Rothman J. The first clinical use of a live-attenuated Listeria monocytogenes vaccine: a Phase I safety study of Lm-LLO-E7 in patients with advanced carcinoma of the cervix. Vaccine. 2009; 27:397583. https://doi.org/10.1016/j.vaccine.2009.04.041.

15. Paterson Y, Guirnalda PD, Wood LM. Listeria and Salmonella bacterial vectors of tumor- associated antigens for cancer immunotherapy. Semin Immunol. 2010; 22:1839. https://doi.org/10.1016/j.smim.2010.02.002.

16. Kruis W, Fric P, Pokrotnieks J, Lukás M, Fixa B, Kascák M, Kamm MA, Weismueller J, Beglinger C, Stolte M, Wolff C, Schulze J. Maintaining remission of ulcerative colitis with the probiotic Escherichia coli Nissle 1917 is as effective as with standard mesalazine. Gut. 2004; 53:1617-23. https:// doi.org/10.1136/gut.2003.037747.

17. Stritzker J, Weibel S, Hill PJ, Oelschlaeger TA, Goebel W, Szalay AA. Tumor-specific colonization, tissue distribution, and gene induction by probiotic Escherichia coli Nissle 1917 in live mice. Int J Med Microbiol. 2007; 297:151-62. https://doi.org/10.1016/j.ijmm.2007.01.008.

18. Maeshima Y, Colorado PC, Torre A, Holthaus KA, Grunkemeyer JA, Ericksen MB, Hopfer H, Xiao Y, Stillman IE, Kalluri R. Distinct antitumor properties of a type IV collagen domain derived from basement membrane. J Biol Chem. 2000; 275:21340-8. https://doi.org/10.1074/ jbc. M001956200.

19. Maeshima Y, Manfredi M, Reimer C, Holthaus KA, Hopfer H, Chandamuri BR, Kharbanda S, Kalluri R. Identification of the anti-angiogenic site within vascular basement membrane- derived tumstatin. J Biol Chem. 2001; 276:15240-8. https://doi.org/10.1074/jbc.M007764200.

20. Hutchings H, Ortega N, Plouët J. Extracellular matrixbound vascular endothelial growth factor promotes endothelial cell adhesion, migration, and survival through integrin ligation. FASEB J. 2003; 17:1520-2. https://doi. org/10.1096/fj.02-0691fje.

21. Kiyuna T, Murakami T, Tome Y, Kawaguchi K, Igarashi K, Zhang Y, Zhao M, Li Y, Bouvet M, Kanaya F, Singh A, Dry S, Eilber FC, Hoffman RM. High efficacy of tumor-targeting Salmonella typhimurium A1-R on a doxorubicin- and dactolisib-resistant follicular dendriticcell sarcoma in a patient-derived orthotopic xenograft PDOX nude mouse model. Oncotarget. 2016; 7:33046-54. https://doi.org/10.18632/oncotarget.8848.

22. Hu CJ, Zhou L, Cai Y. Dihydroartemisinin induces apoptosis of cervical cancer cells via upregulation of RKIP and downregulation of bcl-2. Cancer Biol Ther. 2014; 15:279-88. https://doi.org/10.4161/cbt.27223.

23. Lertkiatmongkol $\mathrm{P}$, Liao D, Mei H, Hu Y, Newman PJ. Endothelial functions of platelet/ endothelial cell adhesion molecule-1 (CD31). Curr Opin Hematol. 2016; 23:253-9. https://doi.org/10.1097/MOH.0000000000000239.

24. Dana A, Wang LM, D'Costa Z, Azad A, Silva MA, Soonawalla Z, Allen P, Liu S, McKenna WG, Muschel RJ, Fokas E. Prognostic role and correlation of CA9, CD31, CD68 and CD20 with the desmoplastic Stroma in pancreatic ductal adenocarcinoma. Oncotarget. 2016; 7:72819-32. https//doi.org/10.18632/oncotarget.12022.

25. Nissle A. Die antagonistische Behandlung chronischer Darmstörungen mit Colibakterien. Med Klin. 1918; 2:29-30.

26. Sonnenborn U, Schulze J. The non-pathogenic Escherichia coli strain Nissle 1917-features of a versatile probiotic. Microb Ecol Health Dis. 2009; 21:122-58. https://doi. org/10.3109/0891060090344 4267.

27. Low KB, Ittensohn M. Lipid A mutant Salmonella with suppressed virulence and TNF alpha induction retain tumortargeting in vivo. Nat Biotechnol. 1999; 17:37-41. https:// doi.org/10.1038/5205.

28. Danino T, Prindle A. Programmable probiotics for detection of cancer in urine. Sci Transl Med. 2015; 7:289ra84. https:// doi.org/10.1126/scitranslmed.aaa3519.

29. Yunlei Z, Youming Z, Liqiu X, Zhang X, Ding X, Yan F, Wu F. Escherichia coli Nissle 1917 targets and restrains mouse B16 melanoma and 4T1 breast tumors through expression of azurin protein. Appl Environ Microbiol. 2012; 78:760310. https://doi.org/10.1128/AEM.01390-12.

30. Sasaki T, Fujimori M, Hamaji Y, Hama Y, Ito K, Amano J, Taniguchi S. Genetically engineered Bifidobacterium longum for tumor-targeting enzymeprodrug therapy of autochthonous mammary tumors in rats. Cancer Sci. 2006; 97:649-57. https://doi. org/10.1111/j.1349-7006.2006.00221.x.

31. Longoria RL, Cox MC, Figg WD. Antiangiogenesis: a possible treatment option for prostate cancer? Clin Genitourin Cancer. 2005; 4:197-202. https://doi. org/10.3816/CGC.2005.n.033.

32. Petersen I. Antiangiogenesis, anti-VEGF(R) and outlook. Recent Results Cancer Res. 2007; 176:189-99. https://doi. org/10.1007/978-3-540-46091-6_16.

33. Li C, Guan X, Sun B, Ma M, Wang P, Gai X. Vectormediated Tum-5 expression in neovascular endothelial cells 
for treating hepatocellular carcinoma. Exp Ther Med. 2017; 13:1521-5. https://doi.org/10.3892/etm.2017.4127.

34. Yanjie Y, Xiaochang X, Meng L, Qin X, Zhang C, Wang W, Giang C, Wu S, Liu Y, Zhu W, Ran Y, Zhang Z, Han W, Zhang Y. Inhibition effect of pcDNA-tum-5 on the growth of S180 tumor. Cytotechnology. 2008; 56:97-104. https:// doi.org/10.1007/s10616-007-9117-9.

35. Cronin M, Akin AR, Collins SA, Meganck J, Kim JB, Baban CK, Joyce SA, van Dam GM, Zhang N, van Sinderen D, O'Sullivan GC, Kasahara N, Gahan CG, et al. High resolution in vivo bioluminescent imaging for the study of bacterial tumour targeting. PLoS One. 2012; 7:e30940. https://doi.org/10.1371/journal.pone.0030940.

36. Wei XW, Zhang ZR, Wei YQ. Anti-angiogenic drugs currently in Phase II clinical trials for gynecological cancer treatment. Expert Opin Investig Drugs. 2013; 22:1181-92. https://doi.org/10.1517/13543784.2013.812071.

37. Shojaei F. Anti-angiogenesis therapy in cancer: current challenges and future perspectives. Cancer Lett. 2012; 320:130-7. https://doi.org/10.1016/j.canlet.2012.03.008.

38. Smith AB 3rd, Freeze BS, LaMarche MJ, Sager J, Kinzler KW, Vogelstein B. Discodermolide analogues as the chemical component of combination bacteriolytic therapy. Bioorg Med Chem Lett. 2005; 15:3623-6. https://doi. org/10.1016/j.bmcl.2005.05.068.

39. Jia LJ, Wei DP, Sun QM, Huang Y, Wu Q, Hua ZC. Oral delivery of tumor-targeting Salmonella for cancer therapy in murine tumor models. Cancer Sci. 2007; 98:1107-12. https://doi.org/10.1111/j.1349-7006.2007.00503.x.

40. Gao L, Zhang L, Hu J, Li F, Shao Y, Zhao D, Kalvakolanu DV, Kopecko DJ, Zhao X, Xu DQ. Down-regulation of signal transducer and activator of transcription 3 expression using vector- based small interfering RNAs suppresses growth of human prostate tumor in vivo. Clin Cancer Res. 2005; 11:6333-41. https://doi.org/10.1158/1078-0432. CCR-05-0148.

41. Ganai S, Arenas RB, Forbes NS. Tumour-targeted delivery of TRAIL using Salmonella typhimurium enhances breast cancer survival in mice. Br J Cancer. 2009; 101:1683-91. https://doi.org/10.1038/sj.bjc.6605403.

42. Zhang L, Gao L, Zhao L, Guo B, Ji K, Tian Y, Wang J, Yu H, Hu J, Kalvakolanu DV, Kopecko DJ, Zhao X, Xu DQ. Intratumoral delivery and suppression of prostate tumor growth by attenuated Salmonella enterica serovar typhimurium carrying plasmid-based small interfering RNAs. Cancer Res. 2007; 67:5859-64. https://doi. org/10.1158/0008-5472.CAN-07-0098.

43. Loeffler M, Le'Negrate G, Krajewska M, Reed JC. Attenuated Salmonella engineered to produce human cytokine LIGHT inhibit tumor growth. Proc Natl Acad Sci U S A. 2007; 104:12879-83. https://doi.org/10.1073/ pnas.0701959104.

44. Baban CK, Cronin M, O'Hanlon D, O'Sullivan GC, Tangney M. Bacteria as vectors for gene therapy of cancer. Bioeng Bugs. 2010; 1:385-94. https://doi.org/10.4161/ bbug.1.6.13146.

45. Gross ML, Somani P, Ribner BS, Raeader R, Freimer EH, Higgins JT Jr. Ceftizoxime elimination kinetics in continuous ambulatory peritoneal dialysis. Clin Pharmacol Ther. 1983; 34:673-80. https://doi.org/10.1038/ clpt.1983.231.

46. Vasey PA, Shulman LN, Campos S, Davis J, Gore M, Johnston S, Kirn DH, O'Neill V, Siddiqui N, Seiden MV, Kaye SB. Phase I trial of intraperitoneal injection of theE1B-55-kd-gene-deleted adenovirus ONYX-015 (d11520) given on days 1 through 5 every 3 weeks in patients with recurrent/refractory epithelial ovarian cancer. J Clin Oncol. 2002; 20:1562-9. https://doi.org/10.1200/ JCO.2002.20.6.1562.

47. Shimada T, Nomura M, Yokogawa K, Endo Y, Sasaki T, Miyamoto K, Yonemura Y. Pharmacokinetic advantage of intraperitoneal injection of docetaxel in the treatment for peritoneal dissemination of cancer in mice. J Pharm Pharmacol. 2005; 57:177-81. https://doi. org/10.1211/0022357055380. 